\title{
Ulinastatin Improves Renal Microcirculation by Protecting Endothelial Cells and Inhibiting Autophagy in a Septic Rat Model
}

\author{
Tian $\mathrm{Li}^{\mathrm{a}} \quad$ Xiaojun Jib Jingfeng Liu ${ }^{\mathrm{b}} \quad$ Xinjie Guo ${ }^{\mathrm{b}} \quad$ Ran Pang $^{\mathrm{b}}$ Haizhou Zhuang ${ }^{\mathrm{b}}$ \\ Lei Dong $^{b}$ Meili Duan ${ }^{b}$ Ang Lia \\ aDepartment of Critical Care Medicine, Capital Medical University Affiliated Beijing Ditan Hospital, Beijing, China; \\ ${ }^{b}$ Department of Critical Care Medicine, Capital Medical University Affiliated Beijing Friendship Hospital, Beijing, China
}

\section{Keywords}

Sepsis-associated acute kidney injury - Microcirculation ·

Ulinastatin · Vascular endothelial-cadherin · Autophagy

\begin{abstract}
Introduction: Increased permeability of the renal capillaries is a common consequence of sepsis-associated acute kidney injury. Vascular endothelial (VE)-cadherin is a strictly endothelial-specific adhesion molecule that can control the permeability of the blood vessel wall. Additionally, autophagy plays an important role in maintaining cell stability. Ulinastatin, a urinary trypsin inhibitor, attenuates the systemic inflammatory response and visceral vasopermeability. However, it is uncertain whether ulinastatin can improve renal microcirculation by acting on the endothelial adhesion junction. Methods: We observed the effect of ulinastatin in a septic rat model using contrast-enhanced ultrasonography (CEUS) to evaluate the perfusion of the renal cortex and medulla. Male adult Sprague Dawley rats were subjected to cecal ligation and puncture and divided into the sham, sepsis, and ulinastatin groups. Ulinastatin $(50,000 \mathrm{U} / \mathrm{kg})$ was injected into the tail vein immediately after the operation. The CEUS was performed to evaluate the renal microcirculation
\end{abstract}

Karger@karger.com www.karger.com/kbr

Karger"

BOPEN ACCESS
(C) 2022 The Author(s)

Published by S. Karger AG, Basel

This is an Open Access article licensed under the Creative Commons Attribution-NonCommercial-4.0 International License (CC BY-NC) (http://www.karger.com/Services/OpenAccessLicense), applicable to the online version of the article only. Usage and distribution for commercial purposes requires written permission. perfusion at 3, 6, 12, and $24 \mathrm{~h}$ after the operation. Histological staining was used to evaluate kidney injury scores. Western blot was used to quantify the expression of VE-cadherin, LC3II, and inflammatory factors (interleukin-1 $\beta$, interleukin-6, and tumor necrosis factor- $\alpha$ ) in kidney tissue, and enzyme-linked immunosorbent assay detected serum inflammatory factors and kidney function and early kidney injury biomarker levels. Results: Compared with the sham group, ulinastatin reduced the inflammatory response, inhibited autophagy, maintained the expression of VE-cadherin, and meliorated cortical and medullary perfusion. Conclusion: Ulinastatin effectively protects the adhesion junction and helps ameliorate the perfusion of kidney capillaries during sepsis by the inhibition of autophagy and the expression of inflammatory factors.

(c) 2022 The Author(s).

Published by S. Karger AG, Basel

\section{Introduction}

Sepsis-associated acute kidney injury (S-AKI) is a common complication in critically ill patients. S-AKI is associated with prolonged hospital stay and huge cost and increases the mortality of patients and risk of developing
Correspondence to:

Ang Li, liang@ccmu.edu.cn 
chronic kidney disease [1-4]. Previous studies have suggested that ischemia-reperfusion (IR) is the main cause of acute kidney injury (AKI) in sepsis. With the development of fluid resuscitation therapy, it has been found that the total blood flow of the kidney remains unchanged or increased in the early stage of sepsis, but AKI may still occur [5]. It is clear that S-AKI is obviously different from ischemic AKI in both the experimental and clinical settings [6]. Several pathophysiological mechanisms have been proposed for S-AKI, such as vasodilatation-induced glomerular hypoperfusion, peritubular capillary network dysregulation, inflammatory reactions, and oxidative stress $[7,8]$. Increasing evidence suggests an integrative physiological compartment where these mechanisms come together to play an important role - the renal microcirculation [9].

Endothelial dysfunction plays a central role in microcirculatory dysfunction during S-AKI [10]. Endothelial cell-to-cell junctions play crucial roles in maintaining intercellular adhesion, transferring intracellular signals, and modulating the contact inhibition of cell growth [11]. Therefore, disruption of endothelial junctions may increase vascular permeability by opening intercellular gaps [12]. Vascular endothelial (VE)-cadherin is a strictly endothelial-specific adhesion molecule that controls the permeability of the blood vessel wall for cells and substances [13]. Autophagy is an evolutionarily conserved mechanism that enables the delivery and direct targeting of cytoplasmic materials to the lysosome for degradation and recycling [14]. In recent years, Jiang et al. [15] proved that autophagy of tubular cells had a renoprotective role in AKI. The results of the study by Matsuda et al. [16] showed that autophagy in glomerular endothelial cells (GEnCs) plays an important role in maintaining the integrity of glomerular capillaries. These experiments showed the importance of autophagy in the kidney.

Ulinastatin, a urinary trypsin inhibitor and a potent multivalent Kunitz-type serine protease inhibitor derived from human urine, has been shown to control a series of proinflammatory mediators and cytokines [17]. Recently, studies have shown that ulinastatin has been widely used as a renal protective drug in patients with septic shock and ischemia $[18,19]$. In S-AKI, the effect of ulinastatin on renal microcirculation perfusion remains unclear.

Microcirculation is a functionally independent network of vessels that encompasses arterioles, venules, and capillaries, with diameters ranging from $5 \mu \mathrm{m}$ to $100 \mu \mathrm{m}$ [20]. The pathophysiology of microcirculation can clarify the intricacies involved in tissue perfusion. Renal dysfunction is also known to be associated with a significant change in perfusion [21]; however, there is no clear conclusion about the perfusion of renal tissue in S-AKI. Contrast-enhanced ultrasonography (CEUS) is a promising technology that has been validated to assess and quantify microcirculation up to capillary perfusion [22]. Contrast agents are microbubbles made of gases embedded within a shell that can function as red blood cell tracers. This technology allows for continuous imaging of the vasculature and blood flow [23]. In this study, we used CEUS to evaluate perfusion of the renal cortex and medulla in a septic rat model.

\section{Materials and Methods}

Experimental Animals

A total of 143 Sprague Dawley rats (body weights, 250-300 g) were used in our experiment, and it was ensured that the number of live rats in each group at each time point was six. All animals and the experiments got the approval of the Bioethics Committee of Beijing Friendship Hospital, Capital Medical University. The rats were housed three per cage in a room with an environment temperature of $21-24^{\circ} \mathrm{C}$ with a $12: 12$-h light-dark cycle and were fed standard rat chow with ad libitum access to water. Rats were made to fast for $12 \mathrm{~h}$ before surgery.

\section{Establishment of the Septic Rat Model and Treatment}

Sepsis was induced by cecal ligation and puncture (CLP), as described previously, to establish a rat model of abdominal infection after surgery [24]. An intraperitoneal injection with $10 \%$ chloral hydrate at $0.03 \mathrm{~mL} / 100 \mathrm{~g}$ for anesthesia was used to maintain spontaneous breathing, and the animal was placed supine on the heated platform that can maintain the temperature at $37^{\circ} \mathrm{C}$. A longitudinal skin midline incision was made with a scalpel, with care being taken not to penetrate the peritoneal cavity, and then small scissors were used to extend the incision and to gain entry into the peritoneal cavity $(3-4 \mathrm{~cm})$. The linea alba was identified, and an intermuscular incision was made. The cecum was ligated at half the distance between the distal pole and the base of the cecum (mid-grade sepsis). Cecal contents were gently pushed toward the distal cecum, and at the time of cecal puncture using 18-gauge needles, a droplet of feces was extruded from both the mesenteric and antimesenteric penetration holes to ensure patency. The cecum was moved into the abdominal cavity, and the peritoneum, fasciae, and abdominal musculature were closed with 4-0 waxcoated braided silk nonabsorbable surgical sutures. Animals were resuscitated by injecting prewarmed normal saline $\left(37^{\circ} \mathrm{C}, 5\right.$ $\mathrm{mL} / 100 \mathrm{~g}$ body weight) subcutaneously to demonstrate the early, hyperdynamic phase of sepsis. Rats were returned to their cages immediately at the end of the surgical procedures where access to water and food was available.

The rats were divided into three groups. In the sepsis group, the steps conducted are described above. In the ulinastatin group, rats were treated with $50,000 \mathrm{U} / \mathrm{kg} / 24 \mathrm{~h}$ of ulinastatin via the tail vein immediately after the operation. In the sham group, the cecum was gently squeezed (no perforation), and the animals were injected with the same volume of $0.9 \%$ saline via the tail vein. 


\section{Hemodynamic Measurements}

The blood pressure and heart rate of rats were measured with a rat tail blood pressure monitor (BP-98A; Softron, Tokyo, Japan). Blood lactic acid was measured using a portable blood lactate meter (YK-Scout, Laubach, Germany).

\section{Assessment of Renal Microcirculation Perfusion}

All the rats were anesthetized with $3 \%$ isoflurane in oxygen before imaging. The rats were placed on the ventral side of the heated imaging platform after anesthesia was induced. Anesthesia was maintained by delivering $1-2 \%$ isoflurane in oxygen through a nose cone during imaging. The Vevo 2100 ultrasound system (Fujifilm VisualSonics Inc., Toronto, Canada) in B-mode was used; the MS-400 probe was placed in the renal artery to measure the width of the renal artery, and the M-mode was used to measure the arterial flow rate. The analysis indices were the renal artery resistance index (RI) and average blood flow velocity. Relevant parameters were set as follows: frequency, $30 \mathrm{MHz}$; power, 100\%; frame rate, 12; gain, $28.0 \mathrm{~dB}$; dynamic range, $60 \mathrm{~dB}$; and depth, $7.07 \mathrm{~mm}$.

The unit was equipped with an MS250 transducer, and a wide beam width setting was used to ensure a low, uniform transmission pressure for all depths. Relevant parameters were set as follows: frequency, $18 \mathrm{MHz}$; energy, $10 \%$; frame rate, 36; contrast gain, 37.0 $\mathrm{dB}$; two-dimensional gain: $18.0 \mathrm{~dB}$; depth, $20 \mathrm{~mm}$; and dynamic range, $35 \mathrm{~dB}$.

The focus range was placed in the middle of the left kidney, and $5 \mathrm{~mL}$ of physiological saline was injected into the ultrasonographic contrast agent (SonoVue lyophilized powder; Bracco, Milan, Italy) according to the manufacturer's instructions and shaken to form a sulfur hexafluoride microbubble suspension. Then, 0.2 $\mathrm{mL} / 150 \mathrm{~g}$ of rat body weight was withdrawn, and a rapid bolus was injected into the tail vein of each rat using a dedicated syringe pump, followed by physiological saline $(0.5 \mathrm{~mL})$. Following the contrast injection, 500 frames were recorded, and the stored dynamic images were analyzed offline using Vevo LAB (VisualSonics, Inc.) and Vevo CQ software (nonlinear amplitude modulation contrast imaging; VisualSonics, Inc.).

The cortical and medullary regions of interest were evaluated at the same depths and positions, as much as possible, over a 0.4$\mathrm{mm}^{2}$ area, and the time intensity curve was plotted. The analysis indicators were the peak enhancement $(\mathrm{PE})$, representative of blood volume, mean transit time (mTT), renal resistive index (RI), and pulse index (PI), representative of blood flow. All parameters were measured three times and averaged.

\section{Kidney Dry-Wet Ratio}

The right kidney was removed $24 \mathrm{~h}$ after CLP and weighed. It was placed into a drying oven at $80^{\circ} \mathrm{C}$ for dehydration for $3 \mathrm{~h}$ and weighed.

\section{Enzyme-Linked Immunosorbent Assay}

Blood samples were collected via the abdominal aorta, and urine samples were collected via the metabolic cage. Creatinine (E02C0629; Blue Gene) and blood urea nitrogen (E02C0697; Blue Gene) in the serum were measured using enzyme-linked immunosorbent assay (ELISA) kits. Neutrophil gelatinase-associated lipocalin (NGAL) was measured using rat NGAL ELISA kits (ab119602; Abcam). S-Thrombomodulin was measured using the Rat Soluble thrombomodulin (sTM) ELISA kit (E02S0222; Blue Gene). Serum levels of tumor necrosis factor (TNF)- $\alpha$ (ab46070; Abcam), inter- leukin (IL)-1ß (ab100767; Abcam), and IL-6 (ab119548; Abcam) were measured using a rat bioactive ELISA.

\section{The CEUS of the Kidney}

This was performed at four time points $(3,6,12$, and $24 \mathrm{~h}$ after surgery). By injecting the contrast agent via the tail vein of the rats in the sham group, the contrast agent rapidly reached the renal cortex, and the medullary echo from the renal sinus along the renal arteries was visible. Eventually, the entire renal cortex and medulla formed a "fireball-like" enhancement and then began to subside, and the cortex subsided last.

\section{Histological Examination and Kidney Paller Score}

The left kidney was isolated and fixed by immersion in $4 \%$ paraformaldehyde, embedded in paraffin, and sectioned at a thickness of $4 \mu \mathrm{m}$. Following hematoxylin and eosin staining, pathological changes in kidney cortical and medullary tissues were examined under a light microscope, and slides were reviewed blindly and scored on a semiquantitative scale to evaluate changes found in acute renal failure.

The Paller score was used to assess the severity of renal tubular injury. Slides were reviewed blindly and scored on a semiquantitative scale. Ten high-power fields of view were randomly selected under a light microscope, avoiding repeated scoring of different convolutions of the same tubule, and renal tubular injury was evaluated according to the Paller score. Higher scores represented more severe damage (maximum score per tubule was 10 ), with points given for the presence and extent of tubular epithelial cell flattening, dilated renal tubules (1 point), brush border injury, shedding ( 1 or 2 points), cytoplasmic vacuolization ( 1 point), interstitial edema (1 point), cell membrane bleb formation, cell necrosis, and tubular lumen obstruction ( 1 or 2 points) [25].

Granulocytes were observed under an optical microscope, and 10 high-power fields of view were selected for each pathological section for counting. In each group, six different rat pathological sections were chosen.

\section{Western Blotting}

The left kidney obtained from each group was frozen in liquid nitrogen and stored at $-80^{\circ} \mathrm{C}$. Protein extraction from tissue samples was performed using the RIPA Lysis Buffer (R0010; Solarbio) and Protease and Phosphatase Inhibitor Cocktail (P002, NCM). In brief, protein samples were electrophoresed on 4-12\% sodium dodecyl sulfate-polyacrylamide gel electrophoresis polyacrylamide gels and then transferred to a PVDF membrane (Merck Millipore, Darmstadt, Germany). The membranes were blocked with 5\% BSA in TBST and incubated with the specific primary antibodies ([VE-cadherin Rabbit $\mathrm{mAb}, \mathrm{ab} 231227$; Abcam]; LC3II [E5Q2K] Mouse mAb [\#83506, CST]), TNF- $\alpha$ (ab205587; Abcam), IL-6 (ab259341; Abcam), and IL$1 \beta$ (ab254360; Abcam) overnight at $4^{\circ} \mathrm{C}$. The dilution of VE-cadherin antibody was $5 \mu \mathrm{g} / \mathrm{mL}$, and dilution of the antibodies including lC3II, TNF- $\alpha$, IL- 6 , and IL- $1 \beta$ was 1:1,000. Mouse Anti- $\beta$ eta actin mAb (TA-09) was used as an internal reference, and the dilution was 1:1,000. The membranes were washed with TBST and then incubated with the secondary antibody (goat anti-rabbit IgG $[\mathrm{H}+\mathrm{L}] \mathrm{HRP}$, [111-035-003; Jackson Immuno-Research]), and the dilution of the secondary antibody was 1:10,000. The membranes were scanned with the Odyssey detection system (Li-COR, Lincoln, NE, USA). Densitometric analysis was performed using ImageJ software (National Institutes of Health, Bethesda, MD, USA). 


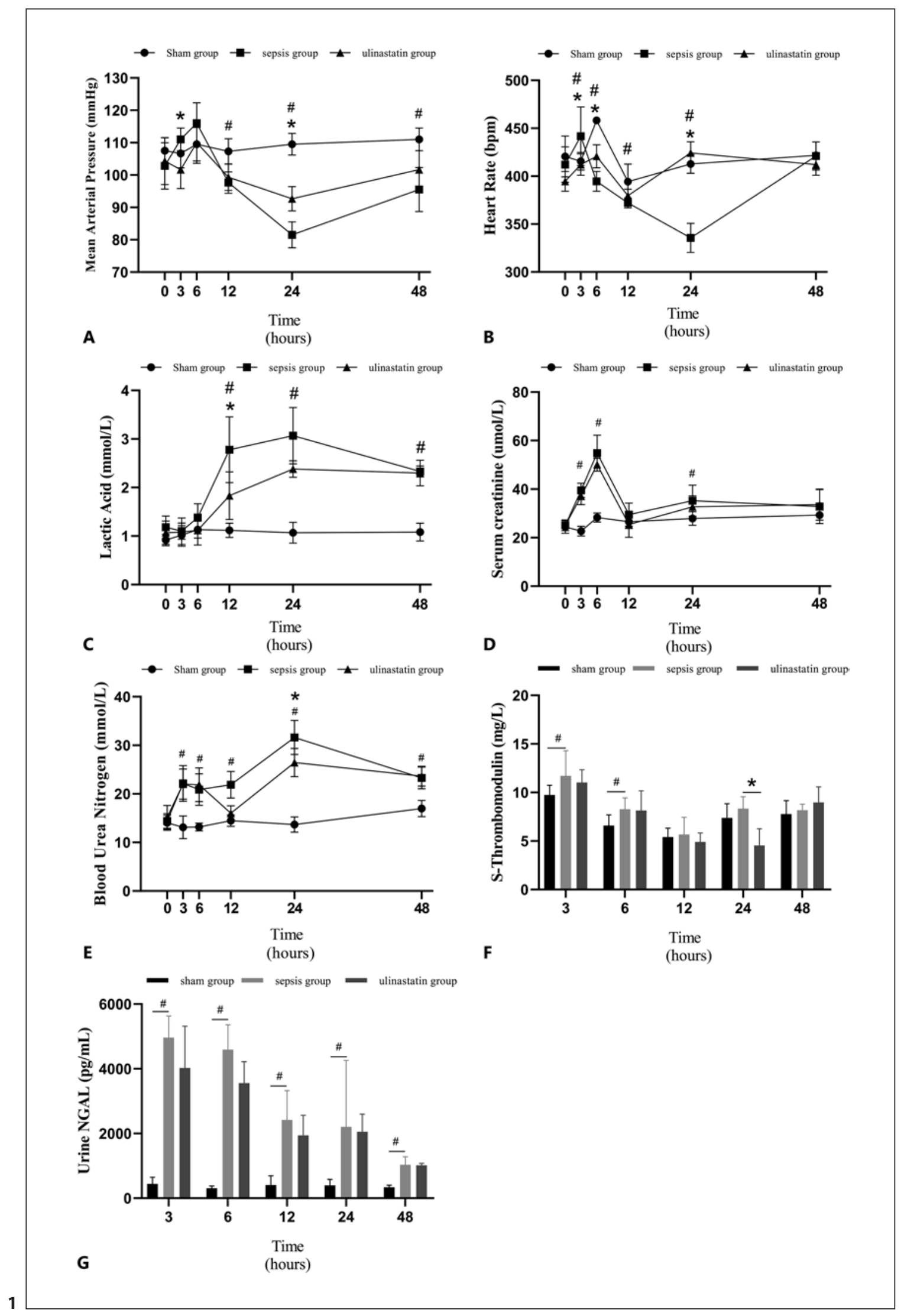

(For legend see next page.) 
Statistical Analysis

Statistical analyses were performed using SPSS 22.0. Data are expressed as the mean $\pm \mathrm{SD}$. After homogeneity test of variances, one-way analysis of variance, followed by multiple comparison of the Tukey test, was used to determine the significance of the different groups. All experiments were conducted at least in triplicate from different cell or tissue samples. Differences were considered significant at $p<0.05$.

\section{Results}

\section{Survival Rates of the Groups}

Rats were observed every $12 \mathrm{~h}$ after the surgery to determine their states and the number of survivors. In the sham group, no rats died at $48 \mathrm{~h}$. The 48 -h mortality rate was $60 \%$ in the sepsis group, and mortality was reduced to $52 \%$ with ulinastatin intervention. Until $72 \mathrm{~h}$, no rats died in the sham group, the mortality in the sepsis group was $76 \%$, and mortality decreased to $68 \%$ in the ulinastatin group (shown in online suppl. Fig. 1; for all online suppl. material, see www.karger.com/doi/10.1159/000521648). However, there was no significant difference between the mortality of the sepsis and ulinastatin groups.

\section{Systemic Hemodynamics and Renal Function}

The mean arterial pressure (MAP) fluctuated between 100 and $110 \mathrm{~mm} \mathrm{Hg}$ in the sham group. In the sepsis group, the MAP had a slight and transient increase (116 $\pm 5 \mathrm{~mm} \mathrm{Hg}$ ) at $6 \mathrm{~h}$, which presented as a state of high dynamics condition (shown in Fig. 1A), and then dropped to $100 \pm 3 \mathrm{~mm} \mathrm{Hg}$ at $12 \mathrm{~h}$. The MAP reached the lowest level ( $82 \pm 4 \mathrm{~mm} \mathrm{Hg})$ at $24 \mathrm{~h}$ in the sepsis group and then began to rise slowly, but it still did not return to the baseline level at $48 \mathrm{~h}$ (shown in Fig. 1A). In the ulinastatin group, the degree of MAP reduction at $24 \mathrm{~h}$ was less than that of the sepsis group $(p<0.05)$. In the sham group, the heart rate increased at $6 \mathrm{~h}$, and the preliminary consideration was that the heart rate changes were related to the day and night rhythms of rats (shown in Fig. 1B). Compared with the sham group, the heart rate of the sepsis

Fig. 1. Systemic hemodynamics and renal function in septic rats. A, B Mean arterial pressure and heart rate values measured at different time points. C Lactate levels based on blood sampling from the tail vein. D-F Creatinine, blood urea nitrogen, and S-thrombomodulin serum levels. G Levels of NGAL based on urine sampling collected through a metabolic cage. NGAL, neutrophil gelatinaseassociated lipocalin. "Sepsis group versus sham group, $p<0.05$, *ulinastatin group versus sepsis group, $p<0.05 . n=6$, at each time point in each group. group increased at $3 \mathrm{~h}$, and it gradually decreased by the next time point, reaching the lowest level at $24 \mathrm{~h}$. Changes in the heart rate were similar to those of the MAP. In the ulinastatin group, the heart rate was similar to that in the sham group (shown in Fig. 1B).

In the sham group, thelactatelevel was $0.8-1.3 \mathrm{mmol} / \mathrm{L}$. There was no difference in the lactate value within $6 \mathrm{~h}$ among the three groups. Lactate levels increased at $12 \mathrm{~h}$ $(2.78 \pm 0.68 \mathrm{mmol} / \mathrm{L})$ and then reached a peak at $24 \mathrm{~h}$ $(3.07 \pm 0.58 \mathrm{mmol} / \mathrm{L})$ in the sepsis group (shown in Fig. 1C). The lactate level in the ulinastatin group was lower than that in the sepsis group at $12(1.83 \pm 0.49$ $\mathrm{mmol} / \mathrm{L})$ and $24 \mathrm{~h}(2.38 \pm 0.17 \mathrm{mmol} / \mathrm{L})$ (shown in Fig. 1C). Figure 1 shows that ulinastatin improved the macroscopic hemodynamics of septic rats.

In the sepsis group, the creatinine level gradually increased at 3-6 h, reaching the peak at $6 \mathrm{~h}(54.83 \pm 7.39$ $\mu \mathrm{mol} / \mathrm{L}$ ), and the increase was considered to be related to mixed factors such as surgery, rehydration, and anesthesia. The creatinine level decreased at $12 \mathrm{~h}(29.48 \pm 4.78$ $\mu \mathrm{mol} / \mathrm{L})$, then gradually increased, and reached its second peak at $24 \mathrm{~h}(35.25 \pm 6.45 \mu \mathrm{mol} / \mathrm{L})$. In the sepsis group, the creatinine level was significantly higher than that of the sham group at 3, 6, and $24 \mathrm{~h}$. After ulinastatin application, the creatinine levels at 6 and $12 \mathrm{~h}$ were lower than those in the sepsis group, but there was no statistical difference in the two groups (shown in Fig. 1D). Blood urea nitrogen increased at $3 \mathrm{~h}$, maintained a plateau between 6 and $12 \mathrm{~h}$, and then reached the peak at $24 \mathrm{~h}$ in the sepsis group (shown in Fig. 1E). Blood urea nitrogen levels were lower in the ulinastatin group both at 12 and $24 \mathrm{~h}$, and there was a significant difference at $24 \mathrm{~h}$ in the sepsis and ulinastatin groups (shown in Fig. 1E). In the sepsis group, S-thrombomodulin (S-TM) levels were higher than those in the sham group, especially at 3 and $24 \mathrm{~h}$. The concentration of S-TM in the serum was significantly reduced at $24 \mathrm{~h}$ in the ulinastatin group (shown in Fig. 1F). The urine neutrophil gelatinase-associated lipocalin (NGAL) level increased most obviously after $3 \mathrm{~h}$ and gradually decreased. Treatment with ulinastatin reduced the expression of NGAL in urine at $3 \mathrm{~h}$ and $6 \mathrm{~h}$ (shown in Fig. $1 \mathrm{G}$ ).

\section{Ulinastatin Reduces the Expression of Inflammatory}

Factors

In the sepsis group, we observed a significant increase in the levels of inflammatory factors. There were differences in the expression of TNF- $\alpha$ in the serum and kidney tissues. In the sepsis group, the expression of TNF- $\alpha$ in the serum and kidney tissue was significantly increased at $3 \mathrm{~h}$. The TNF- $\alpha$ level in the serum gradually decreased 


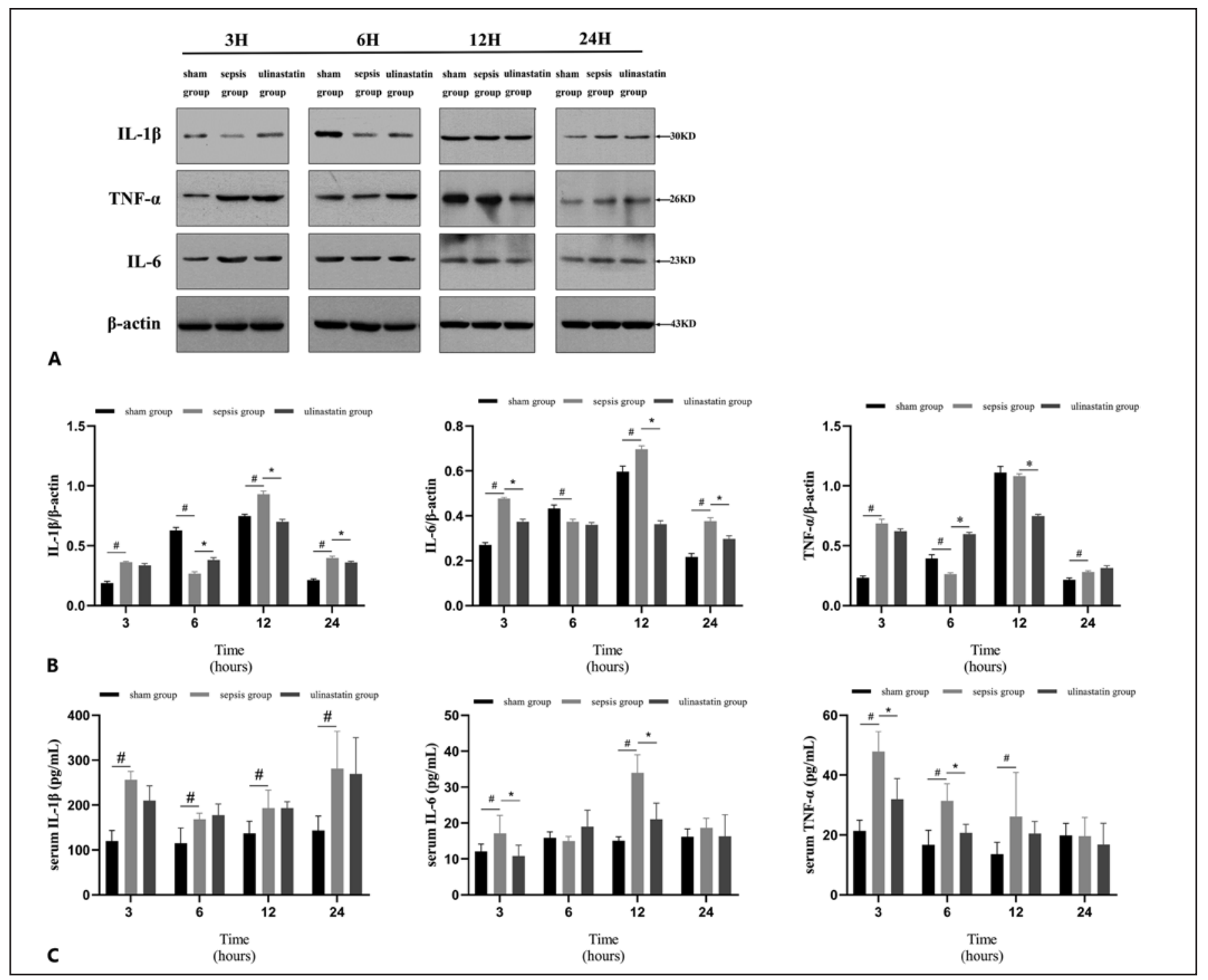

Fig. 2. Inflammatory factors in septic rats at different time points. A, B Western blotting was used to quantify the expression of inflammatory factors in the kidney tissue. C Enzyme-linked immu- nosorbent assay was used to measure the inflammatory factors levels in the serum. "Sepsis group versus sham group, $p<0.05$, *ulinastatin group versus sepsis group, $p<0.05(n=6)$.
Fig. 3. Contrast-enhanced ultrasound images and related parameters. A Renal gray-scale contrast-enhanced ultrasonography showing the real-time contrast-matched imaging technique. Before injecting the contrast agent (a), cortical filling period (b), cortical enhancement period (c), medullary filling period (d), enhanced period (e), and dissipation period (f). B Bolus perfusion model: perfusion quantification represents the core of the Vevo CQ functionality and performs quantification in two steps. (1) Video data are first converted into echo-power data, a quantity directly proportional to the instantaneous concentration of the contrast agent at each location in the field of view; this conversion process is called linearization. (2) The echo-power data as a function of time, or linearized signals, are then processed to assess blood perfusion, using a curve-fitting approach with a parametric perfusion model. $\mathbf{C}$ The intensity before the contrast agent reached the renal parenchyma is set to 0 , and the cumulative intensity is calculated. The intensity of the contrast agent is encoded by a particular color on the parameter color scale. The medulla of the renal cortex is shown in cyan and blue, and the cortex is shown in red and yellow. In the sham group, the boundary between the renal cortex and medulla is noticeable. Abnormal perfusion is observed in the sepsis group, and several renal cortical locales are lost. In the ulinastatin group, cortical perfusion is significantly enhanced. D Parameters related to the left renal artery. E Renal cortical and medullary perfusion-related parameters. TTP, time to peak; mTT, mean transit time; WIR, washin perfusion index; RT, rise time; PE, peak enhancement; PI, pulse index; RI, resistive index. "Sepsis group versus sham group, $p<0.05$, *ulinastatin group versus sepsis group, $p<0.05(n=6)$.

(For figure see next page.) 


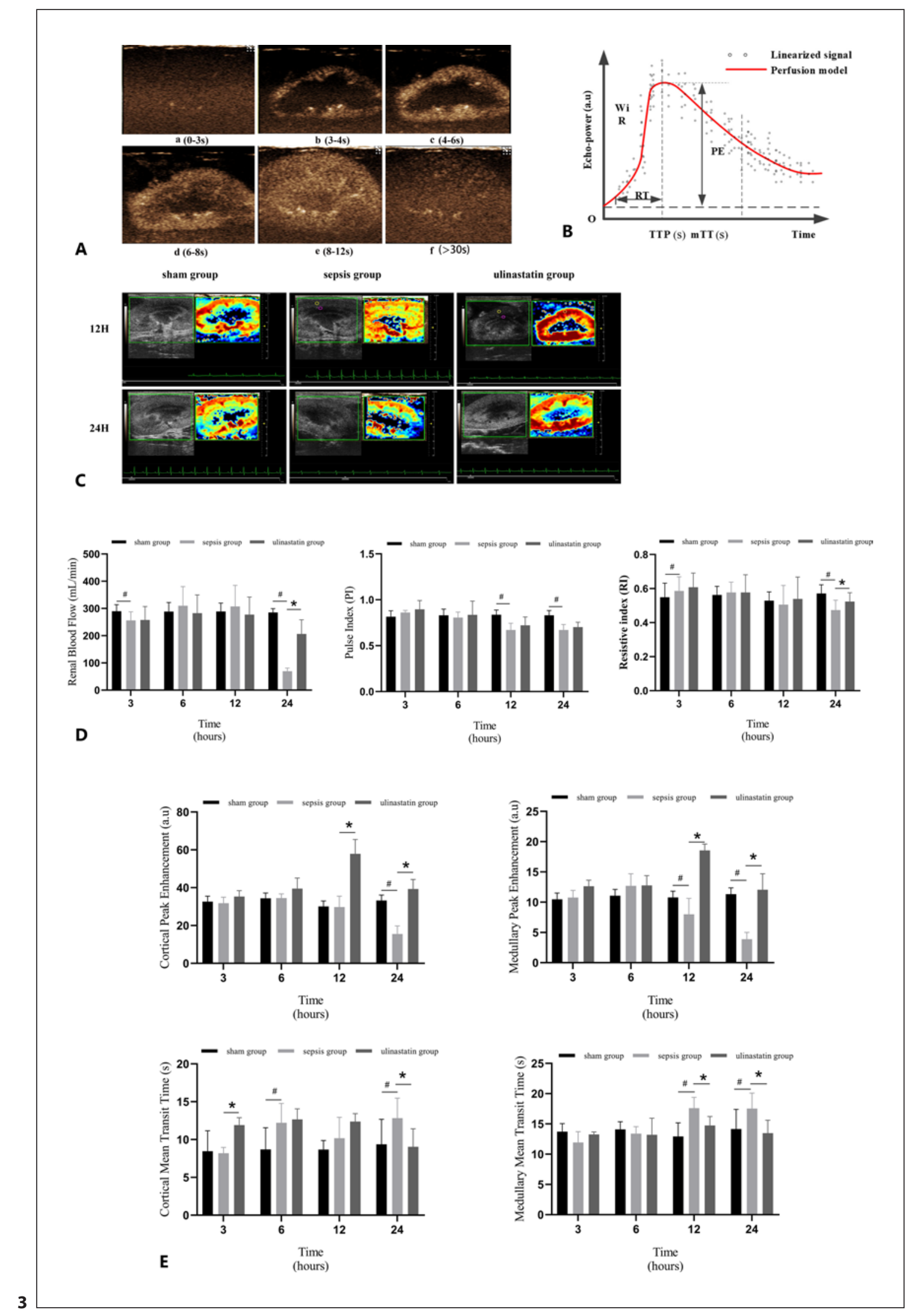


until $24 \mathrm{~h}$, while in the kidney tissues, the TNF- $\alpha$ level showed the second peak at $12 \mathrm{~h}$. Ulinastatin application alleviated the expression of TNF- $\alpha$ in the kidney tissue at $12 \mathrm{~h}$ but did not show the same change in the serum (shown in Fig. 2A-C).

In the sepsis group, IL-6 levels increased significantly at $3 \mathrm{~h}$ and $12 \mathrm{~h}$ in the serum (shown in Fig. 2C). In the kidney tissue, the expression of IL-6 was consistent with its change trend in the serum (shown in Fig. 2B). Treatment with ulinastatin reduced the expression of IL- 6 both in the serum and kidney tissue at 3 and $12 \mathrm{~h}$ (shown in Fig. 2A-C).

In the sepsis group, the IL- $1 \beta$ level in the serum was significantly increased at all time points, especially at 3 and $24 \mathrm{~h}$ (shown in Fig. 2C). IL-1 $\beta$ in the kidney tissue was significantly increased at $12 \mathrm{~h}$, which was different from the change trend of IL- $1 \beta$ in the serum (shown in Fig. 2B). After the application of ulinastatin, there was no significant difference in the expression of IL- $1 \beta$ in the serum, and the expression of IL- $1 \beta$ in the kidney tissue was significantly reduced at $12 \mathrm{~h}$ (shown in Fig. 2A-C).

\section{CEUS of the Kidney}

Figure $3 \mathrm{~A}$ shows the processing of the contrast agent from injection to dissipation, and it could be simply divided into six periods. The normal times for each period in the sham group were as follows: (a) before injecting the contrast agent $(0-3 \mathrm{~s})$; (b) the cortical filling period (3-4 s); (c) the cortical enhancement period (4-6 s); (d) the medullary filling period (6-8 s); (e) the enhanced period $(8-12 \mathrm{~s})$; and (f) the dissipation period (>30 s). Perfusion estimates in Vevo CQ are made by a curve-fitting process that adjusts the parameters of a mathematical model function to best fit the experimental linearized signal. In the context of ultrasound contrast imaging, the mathematical function is called the perfusion model (shown in Fig. 3B). The model serves to estimate a set of perfusion parameters for quantification purposes that can be divided into three categories: amplitude (PE), time (rise time [RT] and mTT), and a combination of amplitude and time (WiR). The intensity before the contrast agent reached the renal parenchyma was set to 0 , and the cumulative intensity was calculated. The contrast agent intensity is encoded by a particular color on the parameter color scale. The rat medulla is shown in cyan and blue, and the cortex is shown in red and yellow (shown in Fig. 3C), illustrating the position of the regions of interest in the renal cortex (continuous yellow line) and renal medulla (continuous red line), avoiding the inclusion of the arteries. The renal artery blood flow (RBF) was calculated by measuring the diameter of the renal artery $(d)$ and the average blood flow velocity (TAV) in the renal artery using the formula: $A=\left(\pi d^{2} / 4\right) \times \mathrm{TAV} . \mathrm{RI}=(\mathrm{PSV}-\mathrm{EDV}) /$ PSV (PSV, peak systolic velocity; EDV, end-diastolic velocity $) . \mathrm{PI}=(\mathrm{PSV}-\mathrm{EDV}) / \mathrm{TAV}$, and TAV $=(\mathrm{PSV}+\mathrm{EDV}$ $\times 2) / 3$, and mainly reflects changes in vascular elasticity and compliance.

In the sepsis group, RBF increased at 6 and $12 \mathrm{~h}$, as shown in Figure 3D, but the MAP began to decrease after $12 \mathrm{~h}$. Therefore, the change in RBF was later than the change in the MAP. We also got the RI and PI of the renal artery. In the sepsis group, the RI gradually decreased and was accompanied by a decrease in the PI (shown in Fig. 3D). After applying ulinastatin, the renal artery RI was improved at $24 \mathrm{~h}$, but PI has not improved significantly.

In the sham group, the outlines of the cortex and medulla were clear, and the cortical and medullary PE values were $32 \pm 3 \mathrm{~dB}$ and $10 \pm 2 \mathrm{~dB}$ (shown in Fig. $3 \mathrm{E}$ ), respectively. The mean velocity of the renal artery was $394 \pm 27$ $\mathrm{mm} / \mathrm{s}$. In the sepsis group at $24 \mathrm{~h}$, a part of the renal parenchyma was not filled with contrast agent, the mean velocity in the renal artery decreased to $161 \pm 49 \mathrm{~mm} / \mathrm{s}$, and the outlines of the cortex and medulla were no longer clear (shown in Fig. 3C). The mean velocity in the renal artery in the ulinastatin group was maintained at $479 \pm$ $149 \mathrm{~mm} / \mathrm{s}$.

Under these conditions, we further measured the blood perfusion of the cortical medulla of the kidney. The trend of the cortical PE change was consistent with the trend of the RBF. Compared with the sham group, PE in the sepsis group showed no obvious change before 12 $\mathrm{h}$, and there was a significant decrease at $24 \mathrm{~h}$ (shown in Fig. 3E). The change of renal medulla blood flow was earlier than that of the cortex, and it began to decrease significantly at $12 \mathrm{~h}$ in the sepsis group. It seemed that medullary perfusion was more related to the MAP, whereas cortical perfusion was related to the RBF (shown in Fig. 3E). The change in the WiAUC/RT was parallel to

Fig. 4. Transmission electron micrograph of the glomerulus, tubules, and target protein expression in the kidney. A a, b In the sepsis group, two separate autophagosomes (red dashed box) and a fusion autophagolysosome (red solid line box) are seen in the tubules. $\mathbf{d}$, e In the ulinastatin group, no recognizable autophagosomes are observed. c, f Partial swelling of glomerular endothelial cells is observed (thick red arrow and black arrow). B-C Quantification of the target protein in the kidney (vascular endothelialcadherin and LC3II). "Sepsis group versus sham group, $p<0.05$, *ulinastatin group versus sepsis group, $p<0.05(n=6)$.

(For figure see next page.)
Ulinastatin Improves Renal

Microcirculation in Septic Rats 


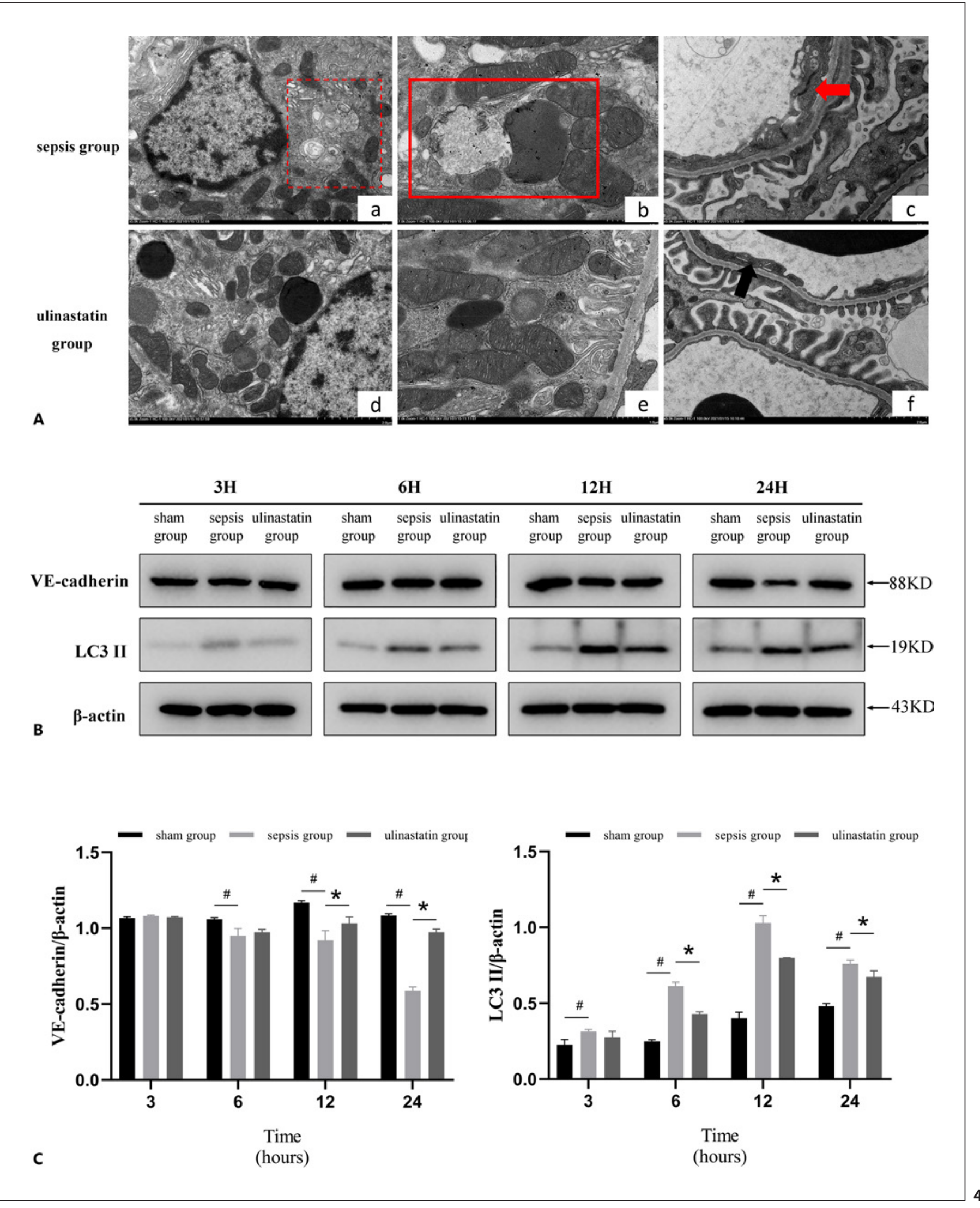




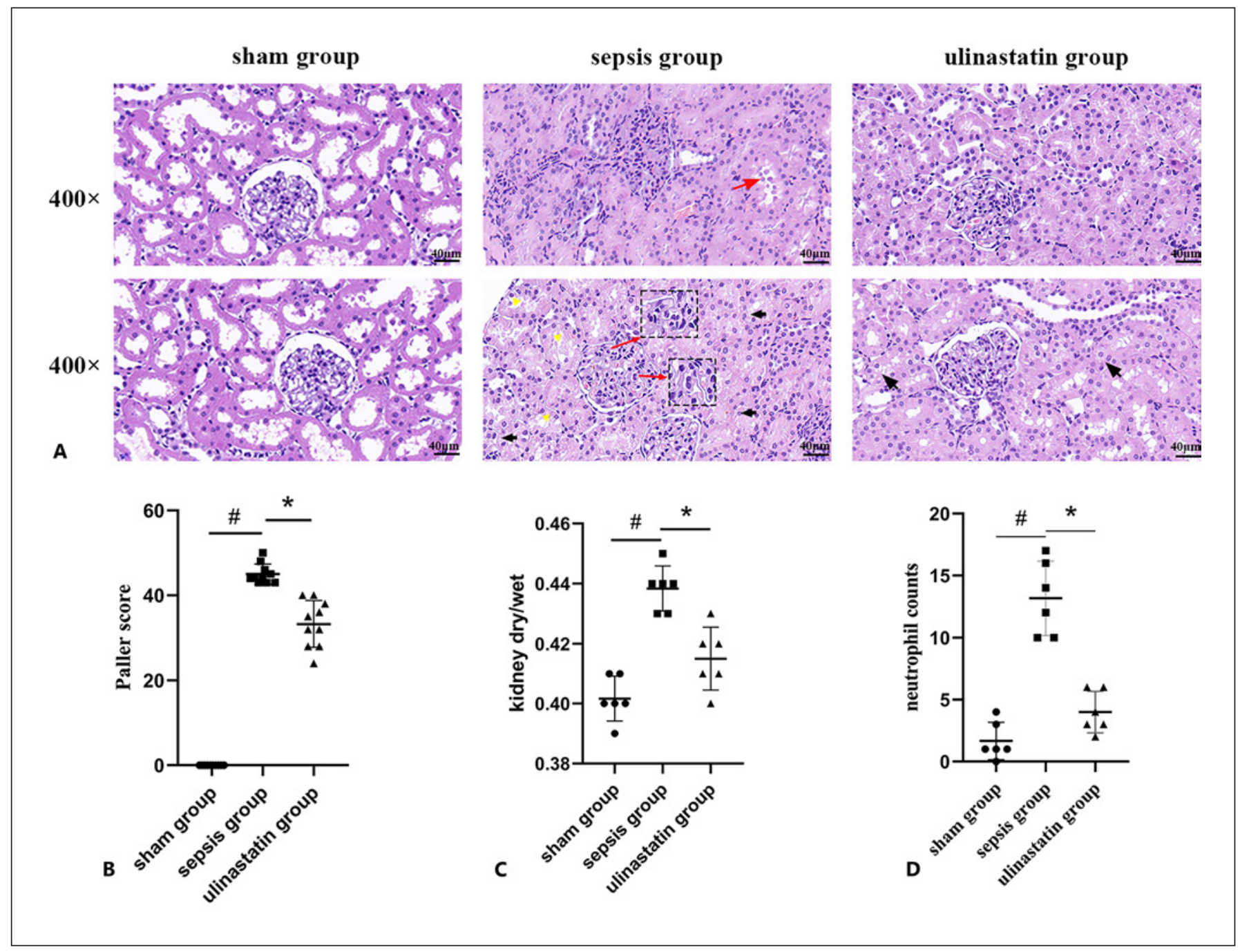

Fig. 5. Histopathology of cecal ligation and puncture-induced AKI at $24 \mathrm{~h}$. A In the sham group, the structure of the glomeruli and tubules is obvious and clear. Erythrocyte stasis in the renal cortex, neutrophils in the renal glomerulus (shown in black dotted box), protein casts in the renal tubules (red arrow), cytoplasmic vacuolization of renal tubular epithelial cells (black arrow), and brush border injury (yellow arrow) can be seen. The change in pathology in the ulinastatin group was mild compared with that in the sepsis group. Hematoxylin-eosin stain, bar, $40 \mu \mathrm{m}$. B The Paller score was lower in the ulinastatin group than in the sepsis group $(p<0.05)$. C Kidney dry-wet weight ratio. D Neutrophil counts. "Sepsis group versus sham group, $p<0.05$, *ulinastatin group versus sepsis group, $p<0.05(n=6)$. that in the PE. The mTT measured the average time that blood takes to transit through a portion of the tissue. Cortical mTT was prolonged first at $6 \mathrm{~h}$ and then returned to the baseline level at $12 \mathrm{~h}$ (shown in Fig. 3E), and we considered that mTT extension at $6 \mathrm{~h}$ may be related to the previous fluid resuscitation. The cortical mTT prolonged at $24 \mathrm{~h}$, which may be related to microvascular injury and decrease of renal perfusion. The medullary mTT was significantly prolonged after $12 \mathrm{~h}$ (shown in Fig. 3E). The average renal artery blood flow rate in- creased and RI improved in the ulinastatin group. At the same time, ulinastatin treatment ameliorated renal microcirculation perfusion at $12 \mathrm{~h}$ and $24 \mathrm{~h}$ in both the cortex and medulla.

\section{Autophagy and Vascular Endothelial-Cadherin Changes in the Kidney}

We observed autophagosomes in the renal tubules and glomerular endothelium with a transmission electron microscope. In the sepsis group, autophagosomes 
were observed in renal tubular epithelial cells, and autophagosomes and lysosomes were fusing (shown in Fig. 4A). We observed the same locations in the ulinastatin group, but no obvious autophagosomes were found (shown in Fig. 4A). We did not observe autophagosomes in GEnCs in both the sepsis group and ulinastatin group, and GEnCs were partially thickened. When autophagy takes place, the cytoplasmic LC3 (i.e., LC3I) enzymatically decomposes a small polypeptide and transforms it into an autophagosome membrane type (i.e., LC3II). Therefore, LC3II could be used to estimate the level of autophagy. We quantified the expression of autophagy in the kidneys using Western blotting. In the sepsis and ulinastatin groups, the expression of LC3II increased (shown in Fig. 4B). At 12 and 24 h, ulinastatin was observed to inhibit the expression of LC3II in the sepsis group, and this finding was significantly different between the sepsis and ulinastatin groups $(p<0.05)$. At the same time, the expression of VE-cadherin was reduced in the sepsis group and reached the lowest level at $24 \mathrm{~h}$. In the ulinastatin group, ulinastatin ameliorated the expression of VE-cadherin during sepsis (shown in Fig. 4B, C).

\section{Histopathology}

At present, there is no uniform definition of pathological changes in S-AKI. In our study, we focused on changes in the glomeruli and tubules. In the sham group, the glomerular structure was clear, and erythrocytes were observed inside some GEnCs. In the sepsis group, the structure of the glomerulus was chaotic, and a large amount of erythrocyte stasis was observed in the glomerulus. Neutrophils were observed in the glomerulus, and a small amount of lymphocyte infiltration was observed in the interstitium. In renal tubular epithelial cells, we observed cytoplasmic vacuolation, brush border injury, and protein casts in the cavity (shown in Fig. 5A).

In the ulinastatin group, erythrocyte stasis in the glomerulus was less than that in the sepsis group. Paller scores were lower in the ulinastatin group than in the sepsis group, and there was a significant difference $(p<$ 0.05 ) (Fig. 5B). The kidney dry-wet weight ratio in the sepsis group was greater than that in the sham group and improved after ulinastatin treatment compared with the sepsis group (shown in Fig. 5C). In the sepsis group, neutrophils exuded significantly in the tissues, especially in the glomeruli. The neutrophil counts in the ulinastatin group were significantly reduced (shown in Fig. 5D).

\section{Discussion}

In this study, we used the typical sepsis model of CLP that closely simulates peritonitis caused by appendix perforation in the clinical setting. Postoperative serum creatinine, urea nitrogen, and urine NGAL increased [26], suggesting that AKI occurred.

The time of AKI occurrence could be divided into two stages: (1) in the first period $(0-6 \mathrm{~h})$, there was a slight increase in the MAP and RBF. TNF- $\alpha$ was significantly increased in this stage. S-TM was significantly increased at $3 \mathrm{~h}$, indicating that endothelial cell damage began in the early stage of sepsis $[27,28]$. The expression of VE-cadherin had not yet changed, and the expression of LC3II was increased. Ultrasonography showed no obvious changes in the renal cortical and medullary blood perfusion. (2) In the second period (12-24 h), the MAP and RBF were decreased, reaching its lowest point at $24 \mathrm{~h}$. Simultaneously, lactic acid levels continued to increase, cortical and medullary perfusion was greatly reduced, and $\mathrm{mTT}$ was prolonged. During this time, IL- 6 and IL$1 \beta$ were gradually increased and S-TM decreased at $12 \mathrm{~h}$ and then gradually increased; the expression of VE-cadherin was continuously decreased, and autophagy was maintained at a high level.

$\mathrm{S}$-AKI is a complex physiological and pathological process, and the hemodynamic states may be different. The observed changes in the heart rate, creatinine level, and urea nitrogen level in our study are similar to a report in rats receiving fluid resuscitation [29]. Controversy always exists regarding changes in the cortex and medulla during S-AKI. A pilot study of post-cardiac surgery AKI showed that cortical perfusion decreased by approximately $50 \%$, but did not report related data on medullary perfusion [30]. In a conscious sheep model of hyperdynamic septic shock with AKI, cortical perfusion was not significantly changed, but medullary perfusion decreased [31]. In our study, cortical and medullary perfusion decreased, but the changes were not synchronized.

VE-cadherin is an important determinant of microvascular integrity in vivo [32]. We found that the deterioration of renal function was accompanied by a decrease in VE-cadherin expression. VE-cadherin is transferred between the cell membrane and cytoplasm through internalization [33], autophagy activates phagocytic ability, and the circulating VE-cadherin vesicles in the cell may be phagocytosed, resulting in a decrease in the expression of VE-cadherin.

Previous studies have proven that ulinastatin can protect endothelial cell and organ functions: (1) ulinastatin 
can inhibit various serine proteases and factors IXa, Xa, Xia, and XIIa [34]; (2) ulinastatin blunts the increase in proinflammatory cytokines and inhibits the secretion of proinflammatory cytokines IL-6 and IL-8 [35]; and (3) ulinastatin downregulates stimulated arachidonic acid metabolism, such as thromboxane B2 production in vitro, by modulating TNF- $a$ production via the inhibition of early growth response factor [36]. In our study, we observed that the renal tissue and serum levels of IL- 6 and TNF- $\alpha$ were obviously decreased in the ulinastatin group. This raised the question of whether the renal protective effects might not be specific to ulinastatin but rather due to the reduced IL- 6 or TNF-a levels. Ozer et al. [37] showed that TNF- $\alpha$ (infliximab) can prevent malondialdehyde elevations in septic lung and kidney tissues and reduce glutathione levels in septic liver, spleen, and kidney tissues. Experiments by Ibrahim et al. [38] reported that IL-6 inhibitors have a protective effect on acute kidney and lung injury caused by sepsis. The above experiments showed that IL- 6 and TNF- $\alpha$ inhibitors can improve sepsis-related multiple organ failure, but did not explain the changes in microcirculation.

Ulinastatin can stabilize the barrier function of the vascular endothelia [39]. Previous studies have shown that ulinastatin inhibits autophagy and exhibits a protective effect against myocardial IR injury [40]. Ulinastatin exerts protective effects against lipopolysaccharide-induced cardiac dysfunction and may be associated with its anti-inflammatory and anti-autophagic activity [41].

At present, it is not clear whether autophagy has protective or harmful effects on AKI. Wu and colleagues [42] demonstrated in experiments that the application of 3-methyladenine, a pharmacological inhibitor of autophagy, can alleviate AKI and protect against LPS-induced AKI. In our study, we found that ulinastatin can inhibit autophagy and play a renoprotective role in S-AKI, suggesting that activation of autophagy may be harmful in sepsis-associated AKI.

In this study, ulinastatin inhibited the expression of inflammatory factors (IL-1 $\beta$, IL-6, and TNF- $\alpha$ ), reducing the activation of endothelial cells, stabilizing the expression of VE-cadherin, and reducing the permeability of endothelial cells. The improvement in kidney dry-wet weight ratio in the ulinastatin group also supports this view.

Our data demonstrate that renal blood flow is preserved after ulinastatin treatment, and we postulated that this is related to VE-cadherin preservation. Despite largescale application of clinical fluid resuscitation treatment strategies, the S-AKI rate has not been effectively reduced. In our study, all animals received fluid resuscitation only once after surgery. The 24-h MAP of the sepsis group was less than that of the ulinastatin group, although there was no significant difference. The VE-cadherin in the renal tissue decreased $6 \mathrm{~h}$ before the change in RBF. The expression of VE-cadherin at each point was consistently higher in the ulinastatin group than in the sepsis group. Thus, we believe that the retention of renal blood flow is related to VE-cadherin retention on the endothelial cell membrane.

This study used CEUS to observe blood perfusion of the renal cortex and medulla during sepsis in rats in real time; we found that changes in RBF are not exactly the same as changes in microcirculation. It may be possible to use this technology to assess renal microvascular blood perfusion in the early stage of sepsis, which can further reduce AKI occurrence. As limitations, this study was only an observational experiment and did not examine the mechanism of the effect of ulinastatin on improving microcirculation. Moreover, this experiment lacked invasive means to monitor dynamics in academic qualifications, and ultrasound results could not be verified. Finally, the small number of experimental animals made it impossible to perform fluid resuscitation based on hemodynamics and simulate the clinical situation.

At present, monitoring of microcirculation blood perfusion and oxygenation in real time still needs to be explored further. Ulinastatin, a potential drug for the treatment of sepsis, plays an important role in inhibiting autophagy expression and verifying factors, and research on the mechanisms of inflammation, autophagy, and microvascular permeability may help to better protect kidney function in S-AKI.

\section{Conclusions}

This study provides specific experimental and ultrasound imaging evidence that ulinastatin is an effective drug that can protect the adherens junctions of endothelial cells and reduce microcirculation dysfunction caused by sepsis. The mechanisms responsible for these effects, at least in part, involve the inhibition of autophagy and the suppression of inflammatory factors during sepsis.

\section{Statement of Ethics}

The experimental scheme was approved by the Bioethics Committee of Beijing Friendship Hospital, Capital Medical University [AEEI-2019-064]. 


\section{Conflict of Interest Statement}

The authors have no conflicts of interest to declare.

\section{Funding Sources}

This work was supported by the Beijing Municipal Natural Science Foundation of China [1192062902].

\section{Author Contributions}

Dr. Ang Li and Meili Duan guided the research and supervised the experiments. Tian Li, Jingfeng Liu, Xinjie Guo, and Ran Pang executed the experiment. Xiaojun Ji, Lei Dong, and Haizhou Zhuang performed histopathological examinations and quality control measures. Tian Li drafted the manuscript.

\section{Data Availability Statement}

The data that support the findings of this study are available from the corresponding author upon reasonable request.

\section{References}

1 Bagshaw SM, Uchino S, Bellomo R, Morimatsu $\mathrm{H}$, Morgera S, Schetz M, et al. Septic acute kidney injury in critically ill patients: clinical characteristics and outcomes. Hong Kong J Nephrol. 2015;17(2):S99.

2 Bouchard J, Acharya A, Cerda J, Maccariello ER, Madarasu RC, Tolwani AJ, et al. A prospective international multicenter study of AKI in the intensive care unit. Clin J Am Soc Nephrol. 2015;10(8):1324.

3 Hoste EAJ, Bagshaw SM, Bellomo R, Cely CM, Colman R, Cruz DN, et al. Epidemiology of acute kidney injury in critically ill patients: the multinational AKI-EPI study. Intensive Care Med. 2015 Aug;41(8):1411-23.

4 Peerapornratana S, Manrique-Caballero CL, Gómez H, Kellum JA. Acute kidney injury from sepsis: current concepts, epidemiology, pathophysiology, prevention and treatment Kidney Int. 2019;96(5):1083-99.

5 Uchino S, Kellum JA, Bellomo R, Doig GS, Morimatsu H, Morgera S, et al. Acute renal failure in critically ill patients: a multinational, multicenter study. JAMA. 2005;294(7): 813-8

6 Bellomo R, Kellum JA, Ronco C, Wald R, Martensson J, Maiden M, et al. Acute kidney injury in sepsis. Intensive Care Med. 2017; 43(3):816-28

7 Haddy FJ. Acute renal failure and sepsis. N Engl J Med. 2004;351(22):2347-9; author reply 47-9.

8 Wu L, Gokden N, Mayeux PR. Evidence for the role of reactive nitrogen species in polymicrobial sepsis-induced renal peritubular capillary dysfunction and tubular injury. J Am Soc Nephrol. 2007;18(6):1807-15.

9 Ergin B, Kapucu A, Demirci-Tansel C, Ince C. The renal microcirculation in sepsis. Nephrol Dial Transplant. 2015;30:169-77.

10 Zafrani L, Payen D, Azoulay E, Ince C. The microcirculation of the septic kidney. Semin Nephrol. 2015;35(1):75-84.

11 Dejana E, Orsenigo F, Molendini C, Baluk P, Mcdonald DM. Organization and signaling of endothelial cell-to-cell junctions in various regions of the blood and lymphatic vascular trees. Cell Tissue Res. 2009;335(1):17-25.
12 Giannotta M, Trani M, Dejana E. VE-cadherin and endothelial adherens junctions: active guardians of vascular integrity. Dev Cell. 2013;26(5):441-54.

13 Vestweber D. VE-cadherin: the major endothelial adhesion molecule controlling cellular junctions and blood vessel formation. Arterioscler Thromb Vasc Biol. 2008;28(2):22332.

14 Schaaf MB, Houbaert D, Meçe O, Agostinis P. Autophagy in endothelial cells and tumor angiogenesis. Cell Death Differ. 2019;26(4): 665-79.

15 Jiang M, Wei Q, Dong G, Komatsu M, Su Y, Dong Z. Autophagy in proximal tubules protects against acute kidney injury. Kidney Int. 2012;82(12):1271-83.

16 Matsuda J, Namba T, Takabatake Y, Kimura T, Takahashi A, Yamamoto T, et al. Antioxidant role of autophagy in maintaining the integrity of glomerular capillaries. Autophagy. 2018 Jan 2;14(1):53-65.

17 Atal SS, Atal S. Ulinastatin: a newer potential therapeutic option for multiple organ dysfunction syndrome. J Basic Clin Physiol Pharmacol. 2016;27(2):91-9.

18 Liu S, Xu J, Gao Y, Shen P, Xia S, Li Z, et al. Multi-organ protection of ulinastatin in traumatic cardiac arrest model. World J Emerg Surg. 2018;13:51.

19 Wang Y, Peng C, Zhang Z, Shi J, Lin Y, Gu L, et al. Intravenous infusion of ulinastatin attenuates acute kidney injury after cold ischemia/reperfusion. Int Urol Nephrol. 2019; 51(25): 1873.

20 Munoz CJ, Lucas A, Williams AT, Cabrales P. A review on microvascular hemodynamics: the control of blood flow distribution and tissue oxygenation. Crit Care Clin. 2020 Apr; 36(2):293-305.

21 Rossi C, Artunc F, Martirosian P, Schlemmer HP, Schick F, Boss A. Histogram analysis of renal arterial spin labeling perfusion data reveals differences between volunteers and patients with mild chronic kidney disease. Invest Radiol. 2012;47(8):490.
22 Harrois A, Duranteau J. Contrast-enhanced ultrasound: a new vision of microcirculation in the intensive care unit. Crit Care. 2013; 17(4):449.

$23 \mathrm{Xu}$ HX, Lu MD. The current status of contrast-enhanced ultrasound in China. J Med Ultrason. 2010;37(3):97-106.

24 Rittirsch D, Huber-Lang MS, Flierl MA Ward PA. Immunodesign of experimental sepsis by cecal ligation and puncture. Nat Protoc. 2009;4(1):31-6.

25 Paller MS, Hoidal JR, Ferris TF. Oxygen free radicals in ischemic acute renal failure in the rat. J Clin Invest. 1984 Oct;74(4):1156-64.

26 Böttiger B, Bernhard M, Knapp J, Nagele P. Influence of EMS-physician presence on survival after out-of-hospital cardiopulmonary resuscitation: systematic review and metaanalysis. Crit Care. 2016;20:4

27 Lin SM, Wang YM, Lin HC, Lee KY, Huang $\mathrm{CD}$, Liu CY, et al. Serum thrombomodulin level relates to the clinical course of disseminated intravascular coagulation, multiorgan dysfunction syndrome, and mortality in patients with sepsis. Crit Care Med. 2008 Mar; 36(3):683-9.

28 Katayama S, Nunomiya S, Koyama K, Wada M, Koinuma T, Goto Y, et al. Markers of acute kidney injury in patients with sepsis: the role of soluble thrombomodulin. Crit Care. 2017; 21(1):229.

29 Arulkumaran N, Sixma ML, Jentho E, Ceravola E, Bass PS, Kellum JA, et al. Sequential analysis of a panel of biomarkers and pathologic findings in a resuscitated rat model of sepsis and recovery. Crit Care Med. 2017; 45(8):e821-30.

30 Schneider AG, Goodwin MD, Schelleman A, Bailey M, Johnson L, Bellomo R. Contrastenhanced ultrasound to evaluate changes in renal cortical perfusion around cardiac surgery: a pilot study. Crit Care. 2013;17(4): R138.

31 Calzavacca P, Evans RG, Bailey M, Bellomo R, May CN. Cortical and medullary tissue perfusion and oxygenation in experimental septic acute kidney injury. Crit Care Med. 2015; 43(10):e431-9. 
32 Corada M, Mariotti M, Thurston G, Smith K, Kunkel R, Brockhaus M, et al. Vascular endothelial-cadherin is an important determinant of microvascular integrity in vivo. Proc Natl Acad Sci U S A. 1999;96(17):9815-20.

33 Basagiannis D, Zografou S, Murphy C, Fotsis $\mathrm{T}$, Morbidelli L, Ziche M, et al. VEGF induces signalling and angiogenesis by directing VEGFR2 internalisation through macropinocytosis. J Cell Sci. 2016;129(22):4091-104.

34 Pugia MJ, Valdes R, Jortani SA. Bikunin (urinary trypsin inhibitor): structure, biological relevance, and measurement. Adv Clin Chem. 2007;44:223-45.

35 Han JI. Urinary trypsin inhibitor: miraculous medicine in many surgical situations? Korean J Anesthesiol. 2010;58(4):325-7.
36 Xu CE, Zhang MY, Zou CW, Guo L. Evaluation of the pharmacological function of ulinastatin in experimental animals. Molecules. 2012;17(8):9070.

37 Ozer EK, Goktas MT, Kilinc I, Toker A, Bariskaner $\mathrm{H}$, Ugurluoglu $\mathrm{C}$, et al. Infliximab alleviates the mortality, mesenteric hypoperfusion, aortic dysfunction, and multiple organ damage in septic rats. Can J Physiol Pharmacol. 2017;95(7):866-72.

38 Ibrahim YF, Moussa RA, Bayoumi AMA, Ahmed A-SF. Tocilizumab attenuates acute lung and kidney injuries and improves survival in a rat model of sepsis via down-regulation of NF- $\kappa B / J N K$ : a possible role of P-glycoprotein. Inflammopharmacology. $2020 \mathrm{Feb}$ 28(1):215-30

39 Wei F, Liu SY, Luo L, Gu NN, Zeng Y, Chen $\mathrm{XY}$, et al. Anti-inflammatory mechanism of ulinastatin: inhibiting the hyperpermeability of vascular endothelial cells induced by TNF- $\alpha$ via the RhoA/ROCK signal pathway. Int Immunopharmacol. 2017;46:220-7.
40 Xiao J, Zhu X, Ji G, Yang Q, Kang B, Zhao J, et al. Ulinastatin protects cardiomyocytes against ischemia-reperfusion injury by regulating autophagy through mTOR activation. Mol Med Rep. 2014;10(4):1949-53.

41 Zhao P, Zhang L. Gao L, Ding Q, Yang Q, Kuai J. Ulinastatin attenuates lipopolysaccharideinduced cardiac dysfunction by inhibiting inflammation and regulating autophagy. Exp Ther Med. 2020;20(2):106472.

42 Wu Y, Wang L, Meng L, Cao GK, Zhao YL, Zhang Y. Biological effects of autophagy in mice with sepsis-induced acute kidney injury. Exp Ther Med. 2019;17(1):316-22. 